\title{
ARCHING OBSERVATION IN THREE-DIMENSIONAL TRAPDOOR PROBLEM WITH X-RAY CT AND DISCRETE ELEMENT METHOD
}

\author{
Bastien Chevalier ${ }^{\text {i) }}$ and Jun Otani ${ }^{\text {ii) }}$
}

\begin{abstract}
A comparative study of the trapdoor problem is proposed in this paper involving experimental model tests on one side and a Discrete Element Method modelling on the other side. Most of the past studies related to the arching effect and the trapdoor problem were performed under plane strain or axisymetric conditions. The observation of the arching mechanisms is indeed simplified under these special test conditions. In this paper, the trapdoor problem was simulated under three-dimensional testing conditions with a square-shaped trapdoor. The granular layers were made with monodisperse glass beads. The arching mechanisms were experimentally investigated with X-ray Computed Tomography. Based on the images obtained, the propagation in the granular layer of the disturbance due to the displacement of the trapdoor was monitored. On the other side, the intensity of the load transfers in the granular material was evaluated with a force measurement apparatus on the trapdoor. In parallel, a numerical analysis involving the Discrete Element Method was conducted to predict both the qualitative and the quantitative phenomena. The gradual local expansion of the granular layer due to trapdoor displacement and the load transfer amplitudes were very well reproduced. An agreement between the experimental and the numerical results was obtained for small displacements of the trapdoor (high load transfers) as well as for larger displacements of the trapdoor.
\end{abstract}

Key words: discrete element method, load transfer, three dimensions, trapdoor, X-ray CT (IGC: E5/E14)

\section{INTRODUCTION}

The arching effect is a universal phenomenon, which takes place in granular materials, among them being soils (Terzaghi, 1936). The arching mechanisms occurring in soils are the key point for determining the pressure applied to buried works, like tunnels and excavations, and also for designing soil reinforcement structures, such as earth structures built on soils prone to any kind of subsidence due to soft soils or sinkholes. The arching mechanisms have been widely studied by means of the trapdoor problem. The trapdoor problem consists of a layer of granular material laying on a support which is able to yield. The yielding part of the support is called the trapdoor. As the trapdoor is moved, arching mechanisms appear in the granular material in the vicinity of the boundary of the trapdoor. The trapdoor's motion can be either upward (passive mode) or downward (active mode). The present study focuses on the active mode. The load transfers resulting from arching mechanisms depend on many parameters of the structures where these mechanisms take place, namely, the height of the granular layer, the size of the trapdoor, the direction of the trapdoor's motion (passive or active mode), the soil characteristics and so on.

The trapdoor problem has been investigated ex- perimentally and theoretically by many researchers. Dewoolkar et al. (2007) listed the major experimental studies on trapdoors with different testing conditions. Most of these studies were performed under plane strain (strip trapdoors) or axisymmetric conditions (circular trapdoors) (Terzaghi, 1936; Tanaka and Sakai, 1993; Vardoulakis et al., 1981; Dewoolkar et al., 2007). In parallel, trapdoors have also been studied with numerical methods such as Finite Element Methods and Discrete Element Methods (Koutsabeloulis and Griffiths, 1989; Tanaka and Sakai, 1993; Chevalier et al., 2009). Finally, based on experimental and numerical observations, many theoretical approaches have been developed for predicting the intensity of load transfers. Some are based on the friction of the whole yielding column of the granular material (Terzaghi, 1943; Handy, 1985), while others are linked to the theory of shear banding (Vardoulakis et al., 1981; Papamichos et al., 2001; Vardoulakis et al., 2004).

Even if observations of trapdoor tests under plane strain conditions can be extended to non-plane strain conditions, it is important to note that most of the arching phenomena occurring in real geotechnical earth structures correspond to non-plane strain conditions. The manifestations of the trapdoor effect in real works are very local in most cases, namely, sinkholes, tunnel face failures and piled embankments. Consequently, ex-

i) Visiting Scholar, X-Earth Center, Kumamoto University, Japan.

ii) Professor, X-Earth Center, Graduate School of Science and Technology, Kumamoto University, Japan (junotani@kumamoto-u.ac.jp). The manuscript for this paper was received for review on February 22, 2010; approved on April 12, 2011.

Written discussions on this paper should be submitted before January 1, 2012 to the Japanese Geotechnical Society, 4-38-2, Sengoku, Bunkyoku, Tokyo 112-0011, Japan. Upon request the closing date may be extended one month. 
perimental studies on the trapdoor problem under nonplane strain conditions are very important. The difficulty of observing the mechanisms occurring during trapdoor tests performed under conditions other than plane strain conditions is the reason why experimental trapdoor problems in three dimensions have not been investigated. The challenging point is to observe the arching mechanisms despite the three-dimensional testing conditions.

In this study, experimental trapdoor tests for the active mode in three dimensions are conducted with a squareshaped trapdoor. The use of the X-ray Computed Tomography (CT) technique makes it possible to observe the arching mechanisms occurring as the trapdoor is moved downward, without altering the three-dimensional aspect of the tests. Glass beads are used to compose the granular layers tested. All the experimental results are compared with the numerical results obtained with the Discrete Element Method (DEM). It is noted that the exact conditions of the experimental tests are reproduced with the numerical model, namely, the model size, the characteristics of the granular materials and the testing process.

\section{METHODS}

\section{Experimental Apparatus}

An industrial X-ray CT scanning system (Otani et al., 2000 , 2002) at Kumamoto University in Japan was used in this series of tests. Consequently, and in order to improve the CT results, an acryl cylindrical container was used to contain the granular layers. This container, shown in Photo 1 and Fig. 1, had an inside diameter of $240 \mathrm{~mm}$ and was supported by a horizontal steel plate under which the trapdoor displacement device was placed. The trapdoor hole, $40 \mathrm{~mm} \times 40 \mathrm{~mm}$, was created in the acryl bottom plate and placed inside the container on the steel plate.

The trapdoor itself, shown in Fig. 2, was an aluminium alloy plate, $40 \times 40 \mathrm{~mm}$ size in plan and $5 \mathrm{~mm}$ in thickness. It was equipped with a steel support located below by means of four parallel vertical steel rods with no play for the steel support. Finally, the steel support was fixed on the displacement control device. The displacement control device was a vertically extendable apparatus consisting of two blocks, moving one from the other with a rack. The rack was moved manually by a screw. The value of the trapdoor displacement, $\delta$, was controlled with a gauge inserted between the two blocks. Considering $\delta=0 \mathrm{~mm}$ as the initial position of the trapdoor (trapdoor at the same level as the container bottom plate), the trapdoor was moved downward once following different displacement steps, namely, $\delta=0.1 \mathrm{~mm}, 1.0 \mathrm{~mm}, 2.0$ $\mathrm{mm}, 5.0 \mathrm{~mm}, 10.0 \mathrm{~mm}$ and $20.0 \mathrm{~mm}$.

Two kinds of experimental tests were distinguished. The first group of tests was dedicated to X-ray CT scanning for which details are given in the following paragraph, while the second group of tests was dedicated to the measurement of the force on the trapdoor in order to evaluate the load transfers occurring in the granular lay-

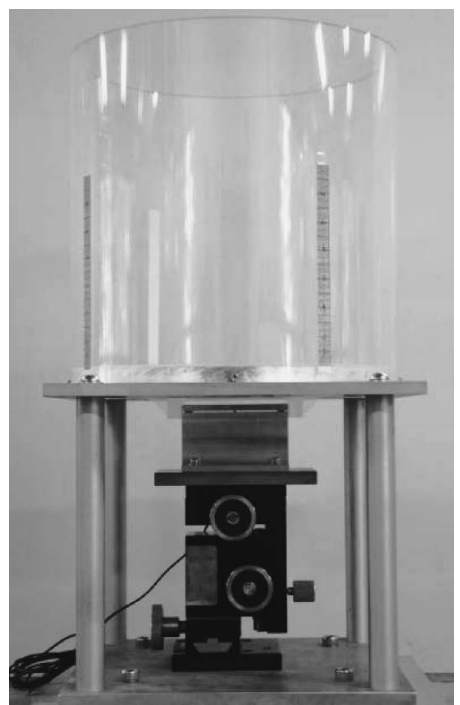

Photo 1. Picture of trapdoor apparatus

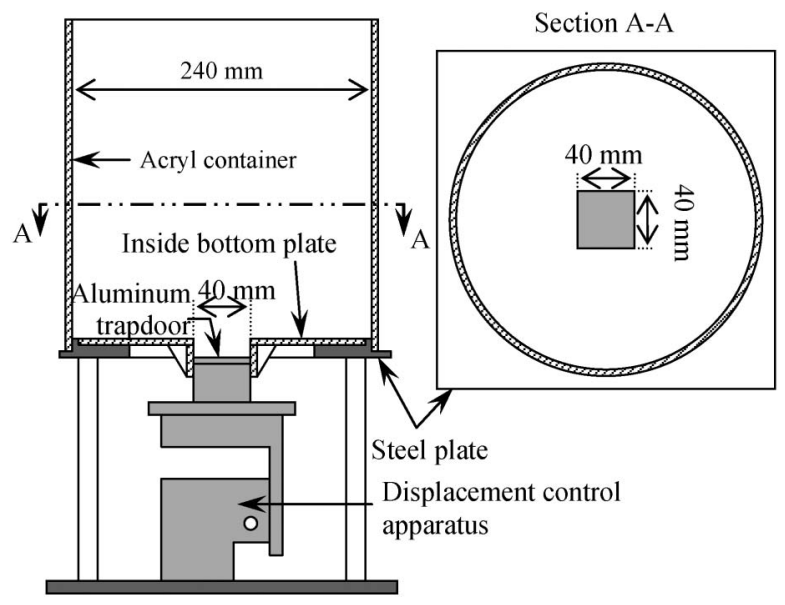

Fig. 1. Configuration of trapdoor apparatus

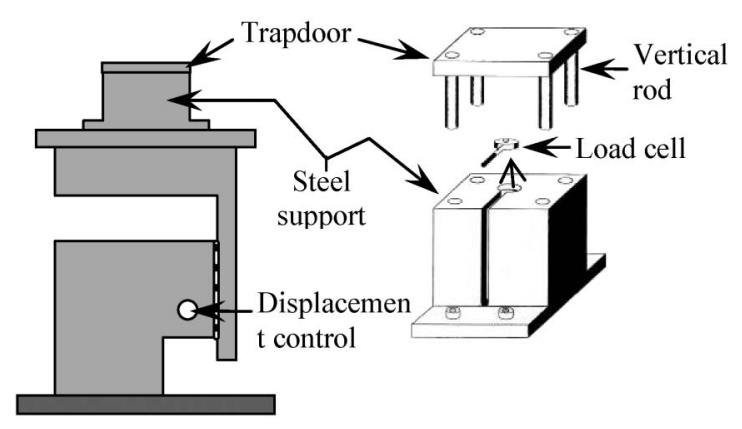

Fig. 2. Configuration of trapdoor

er. The force applied by the granular material on the trapdoor was measured with one load cell located between the trapdoor and its steel support in a centred position, which is shown in Fig. 2. Four vertical rods were fixed under the trapdoor and made it slide vertically into the steel support. The vertical rods kept the trapdoor parallel to its support and prevented all friction that could have oc- 


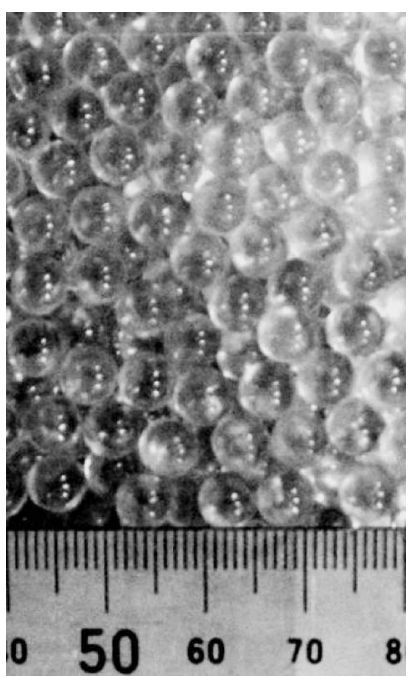

Photo 2. Material of glass beads

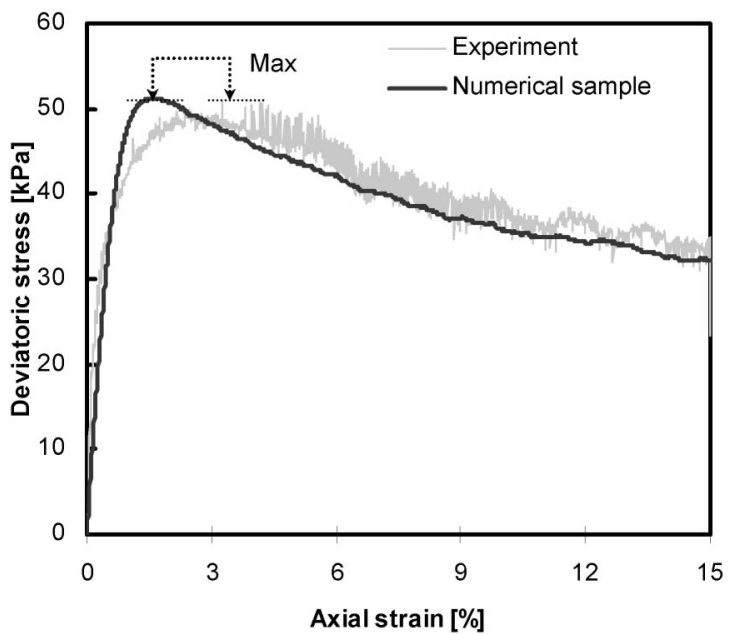

Fig. 3. Triaxial compression tests on glass beads and corresponding numerical modelling

and produced in the sample with a vacuum pump. Friction angle values of $\varphi_{\mathrm{p}}=30^{\circ}$ and $\varphi_{\mathrm{r}}=24^{\circ}$ were obtained. The response of the glass bead samples to the triaxial compression tests is given in Fig. 3 .

Before the trapdoor tests, the glass beads were placed in the container with successive layers of thickness, 20 $\mathrm{mm}$ each. The height of the glass bead samples was finally equal to $80 \mathrm{~mm}$. Due to the very narrow range in void ratio that characterized the glass beads used here, it is noted that no particular densifying method was needed for the sample.

\section{$X$-Ray $C T$}

An X-ray CT is a non-destructive method of investigating the spatial distribution of the density in a material. In this process, a collimated X-ray beam crosses the specimen in several directions by rotation and translation of the specimen. The intensity of the X-ray beams after the penetration of the specimen is computed in order to reconstruct an image of a section of the specimen. The image obtained gives the distribution of the density in the specimen by means of CT values. The CT value links the absorption coefficient of the X-ray beam for scanning point $\mu_{\mathrm{t}}$ to that for water, $\mu_{\mathrm{w}}$, by the following relation:

$$
\mathrm{CT} \text { value }=\left(\mu_{\mathrm{t}}-\mu_{\mathrm{w}}\right) K / \mu_{\mathrm{w}}
$$

where $K(K=1000)$ is a constant number. It can be deduced from this relation that the CT value corresponding to air is -1000 , since the absorption coefficient for air is $\mu_{\mathrm{t}}=0$, while the $\mathrm{CT}$ value corresponding to water is equal to 0 . The relation between the $C T$ value and the density of the material at the scanning point is linear, so that the CT value gives the distribution of the density of a specimen in the cross section. The X-ray CT system used here was equipped with $300 \mathrm{kV}$ X-ray tubes (TOSCANER - 23200 min TOSHIBA Corp., Kumamoto University, Japan).

After preparing the glass bead samples in the container, the whole container was fixed on the turntable of the 


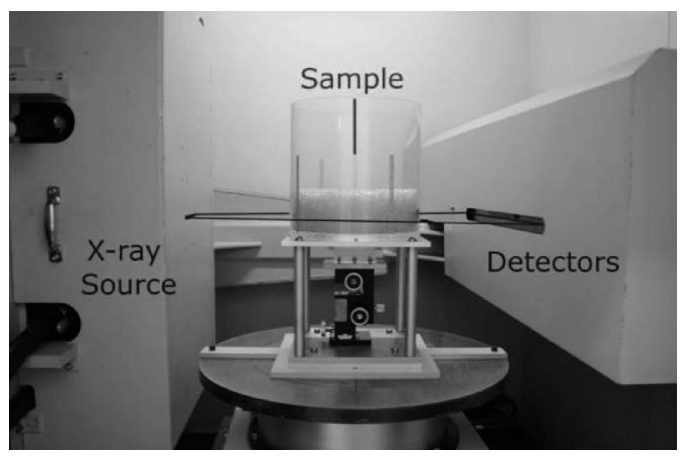

Photo 3. Picture of sample placed in X-ray CT scanner

scanner, as shown in Photo 3. Then, the specimen was scanned with an X-ray beam with an attenuation width of $1 \mathrm{~mm}$ and in a limited region of interest in diameter, namely, $150 \mathrm{~mm}$, in the central part of the container. The total number of voxels in each CT image obtained was $2048 \times 2048$, so that the size of a single voxel of the CT image was equal to $73.2 \times 73.2(\mu \mathrm{m})^{2}$ in plan and $1000 \mu \mathrm{m}$ in height. The specimen was scanned in different states corresponding to different displacement steps, namely, $\delta$ $=0 \mathrm{~mm}$ (initial state), $\delta=2 \mathrm{~mm}, \delta=5 \mathrm{~mm}$ and $\delta=10$ $\mathrm{mm}$.

\section{Discrete Element Method}

The numerical modelling for the trapdoor problem was conducted with DEM based on molecular dynamics (Cundall and Strack, 1979). This method is used in three dimensions with a code called SDEC (Magnier and Donze, 1997) and considers the material as a collection of non-deformable bodies which interact with other bodies through their contact points. Obviously, the particles used here were spherical particles. The normal and tangential contact laws between particles were linear ones, with normal stiffness $k_{\mathrm{n}}$ and tangential stiffness $k_{\mathrm{t}}$. A Coulomb friction criterion of coefficient $\mu$ was used to bind the intensity of the tangential contact force with respect to the normal contact force, as shown in Fig. 4.

The mechanical parameters of the contacts were determined by an inverse analysis in triaxial compression tests. The samples used for the triaxial compression and trapdoor problem tests were prepared by inflating particles without any gravity force or friction (Chareyre and Villard, 2005) in a cylindrical box until the target void ratio of $e=e_{\min }=0.562$ was reached. During this process, due to the expansion of the particles, stress was applied on the wall by the inflated particles. This stress level was limited to a value close to the stress level observed in the sample before the trapdoor tests. Consequently, no change in the void ratio was observed as the gravity forces were applied. More details on the DEM used herein can be obtained in the reference by Chevalier et al. (2009).

\section{Determination of the Model Parameters}

In order to obtain an overlap between particles in the numerical method similar to the normal deformation of the contact point in reality, normal stiffness $k_{\mathrm{n}}$ was deter-

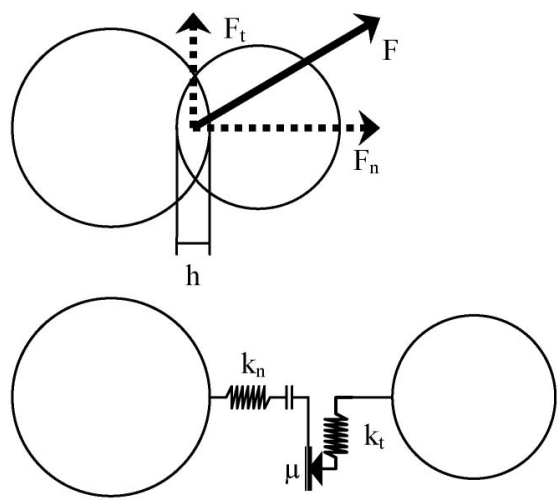

Fig. 4. Discrete element method contact laws

mined based on the stiffness level, $\kappa$ (Combe, 2002). $\kappa$ is a non-dimensional parameter that is inversely proportional to the deflection observed at the contact points. Thornton and Sun (1993) evaluated this coefficient for glass beads as $\kappa=5600$. In the DEM, with a linear normal contact law, the stiffness level of grains can be written (Combe, 2002) as

$$
\kappa=k_{\mathrm{n}} /(\mathrm{d} P)
$$

where $k_{\mathrm{n}}$ is the normal stiffness of the contact, $\mathrm{d}$ is the mean diameter of the particles and $P$ is the pressure level applied to the particle sample. Considering the void ratio, $e=e_{\min }=0.562$, the glass density, $\gamma_{\mathrm{g}}=2.52 \mathrm{~g} / \mathrm{cm}^{3}$, and the height of the granular layer, $h=80 \mathrm{~mm}$, the pressure level applied to the particles during the tests was estimated at around $P=1.5 \mathrm{kPa}$. The normal stiffness was then set to $k_{\mathrm{n}}=6 \times 10^{4} \mathrm{~N} / \mathrm{m}$ which corresponds to $\kappa=6700$. This normal stiffness guaranteed an overlap between the particles in the modelling, not greater than in the experimental tests, even in some areas of concentrated stress.

The tangential stiffness, $k_{\mathrm{t}}$, was defined as the ratio of normal stiffness $k_{\mathrm{n}}$. Combe (2002) showed that considering a ratio $k_{\mathrm{t}} / k_{\mathrm{n}}$ between 0.5 and 1 does not influence the results of the modelling. In addition, Schäfer et al. (1996) recommended setting ratio $k_{\mathrm{t}} / k_{\mathrm{n}}$ to 0.75 , which was considered in the study.

Friction coefficient $\mu$ of the contact law between particles was determined by an inverse analysis in triaxial compression tests on 2-mm diameter spheres presenting a void ratio of $e=e_{\min }=0.562$ and a confining pressure of $25 \mathrm{kPa}$. All the other parameters, such as the particle size distribution, the void ratio, the normal stiffness and the tangential stiffness, were already set. The confining pressure in the triaxial compression tests was higher than the stress level used to set the value of $k_{\mathrm{n}}$. However, the peak deviatoric stress used to set the coefficient, $\mu$, is not influenced by the stiffness level in the range considered here. Friction coefficient $\mu$ was set to $\mu=\tan \left(40^{\circ}\right)$, so that the peak friction angle obtained with the numerical sample was $\varphi_{\mathrm{p}}=30^{\circ}$ and the critical friction angle was $\varphi_{\mathrm{r}}=24^{\circ}$. The response of the numerical sample to the triaxial compression tests is shown in Fig. 3. All the model parameters could be set from the response of the samples in terms of stress only. The dilatancy behaviour of the 


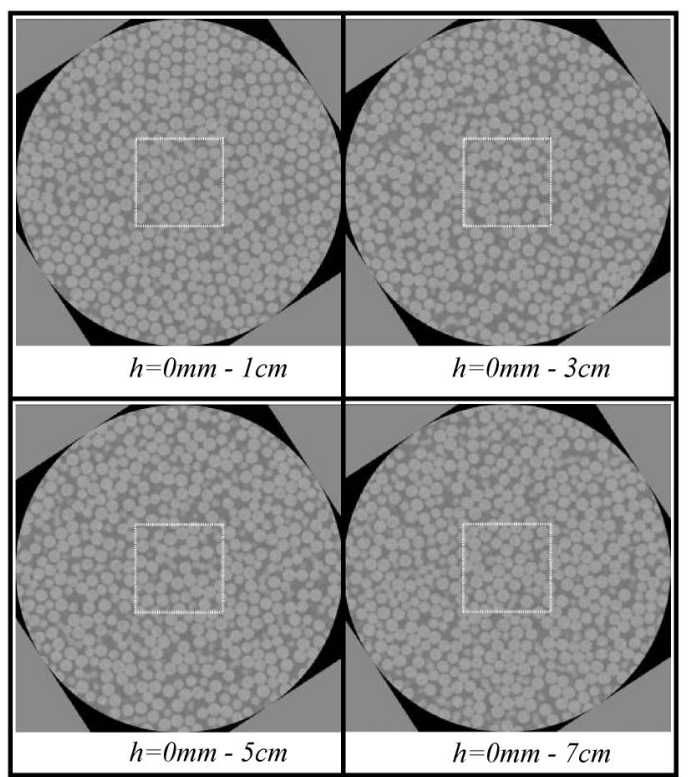

Fig. 5. Raw CT values images for initial state at different heights from bottom of container

sample in the triaxial compression tests was not used here.

In order to evaluate the dispersion of the results obtained with the numerical analysis, three-dimensional trapdoor tests were performed under the same testing conditions.

\section{ANALYSIS OF CT IMAGES}

\section{Binary Images}

The conditions of the CT scanning gave a voxel size equal to $73.2 \times 73.2 \mu \mathrm{m}$ in plan and a height of $1000 \mu \mathrm{m}$. Consequently, one single particle, $6 \mathrm{~mm}$ in diameter, is much bigger than the voxel size; one bead covers around 16,000 voxels. Particles could then be identified on the CT images. A CT value, depending on the density at this point, is associated with each voxel. Each CT value is associated with a grey value, and a total of 256 grey values are used to represent the distribution of density throughout the sample. Figure 5 shows the horizontal cross sections obtained after scanning the sample in its initial state for $\delta=0 \mathrm{~mm}$ and at different heights, h, from the bottom of the container. In our case, the specimen was made of glass beads and air; however the CT values obtained from all the cross sectional images corresponding to the initial state shown in Fig. 6, covered a much wider range than only the two single CT values corresponding to air with a CT value of -1000 and glass with an approximate CT value of 1520 .

The tested samples were binary in their composition. At each point, the material is either air or glass. Consequently, a threshold CT value is determined to separate the voids from the glass beads. The CT image is made of 256 levels of gray, each one corresponding to a CT value interval, called class. Each class $i, i=\{1,2 \ldots, 256\}$ is associated with a median $\mathrm{CT}$ value, $C T_{\mathrm{i}}$. Figure 6 shows the

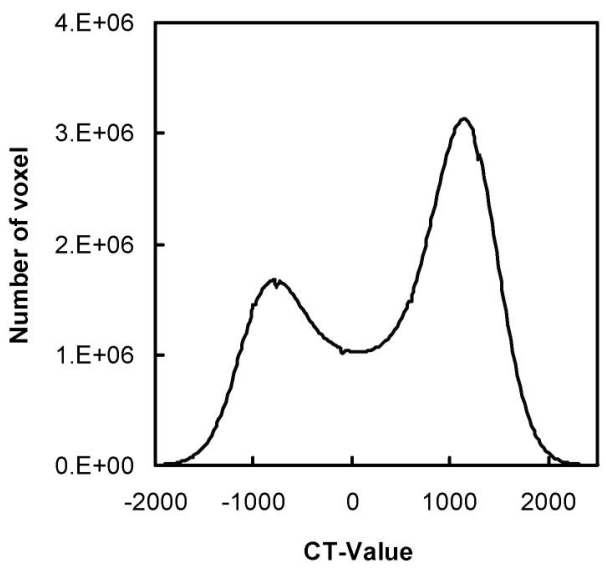

Fig. 6. CT values obtained with $6 \mathrm{~mm}$ glass beads

number of voxels, $n_{\mathrm{i}}$, corresponding to each class, $i$. The total volume $V_{\mathrm{t}}$ of the scanned volume can be calculated by

$$
V_{\mathrm{t}}=v \sum_{i=1}^{256} n_{\mathrm{i}}
$$

where $v$ is the volume of one voxel.

Let $k$ be the class corresponding to the threshold CT value, namely, $C T_{\mathrm{k}}$. The volume of the glass, $V_{\mathrm{g}}$, and the volume of the air, $V_{\mathrm{a}}$, can be calculated.

$$
\begin{aligned}
& V_{\mathrm{a}}\left(C T_{\mathrm{k}}\right)=v \sum_{i=1}^{k} n_{\mathrm{i}} \\
& V_{\mathrm{g}}\left(C T_{\mathrm{k}}\right)=v \sum_{i=k}^{256} n_{\mathrm{i}}
\end{aligned}
$$

Then, void ratio $e$, corresponding to threshold value $C T_{\mathrm{k}}$, can be calculated as

$$
e\left(C T_{\mathrm{k}}\right)=\frac{V_{\mathrm{a}}\left(C T_{\mathrm{k}}\right)}{V_{\mathrm{g}}\left(C T_{\mathrm{k}}\right)}=\frac{\sum_{i=1}^{k} n_{\mathrm{i}}}{\sum_{i=k}^{256} n_{\mathrm{i}}}
$$

The void ratio, $e\left(C T_{\mathrm{k}}\right)$, was calculated for each class $k$ $=\{1,2 \ldots, 256\}$ with Eq. (9). Figure 7 shows the evolution of void ratio $e$ depending on threshold CT value $C T_{\mathrm{k}}$. Knowing the experimental value of the void ratio, $e=e_{\min }$ $=0.562$, the real threshold value, $C T_{\text {th }}$, can be determined as shown in Fig. 7. The threshold CT value corresponding to the void ratio, $e=e_{\min }$, is equal to $C T_{\text {th }}=$ 61.7. Using this threshold value, binary images of the horizontal cross sections of the sample are shown in Fig. 8 in which the black colour shows the air voids and the white colour shows the glass beads.

\section{Comparing the Sample for Different Displacement Steps,} $\delta$

Once binary images are obtained, the glass beads and the voids between them can be separated. The next step is to compare the sample in two different configurations, each configuration corresponding to one value of the displacement of the trapdoor, $\delta$.

Due to similarities in shape and size of the glass beads, 
they cannot be singly identified from one configuration to another. Consequently, the displacement of each glass bead could not be monitored. However, a comparison

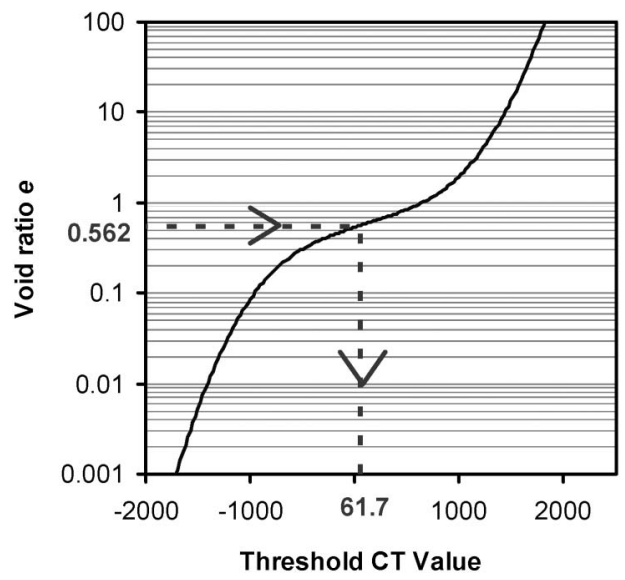

Fig. 7. Void ratio $e$ depending on threshold CT-value

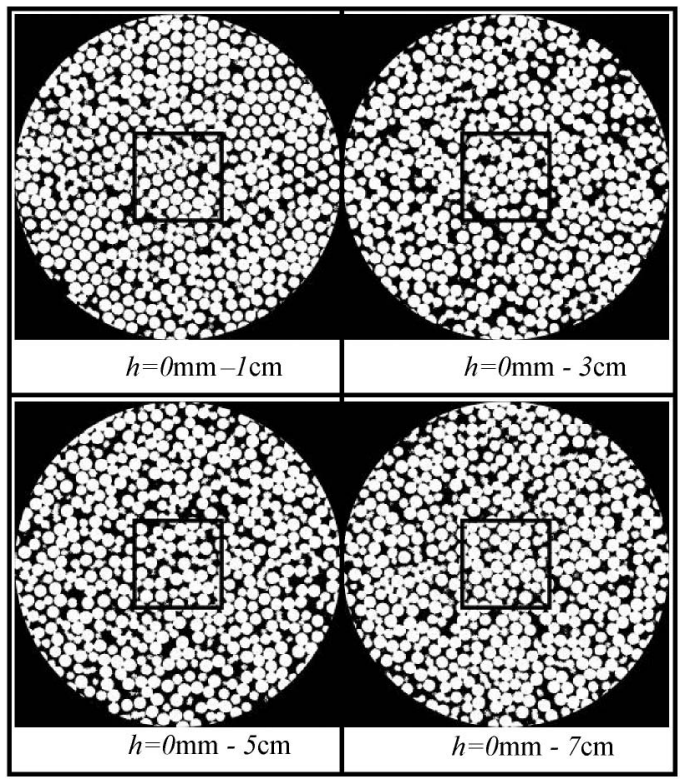

Fig. 8. Binary images of sample in initial state obtained with threshold CT value of 61.7 at different heights from bottom of container process for two configurations is used to differentiate the areas of the sample where groups of beads are moving from the areas where beads remain fixed.

Initial configuration $\mathrm{I}$ and final configuration $\mathrm{F}$ are compared. Let us call $C_{\mathrm{I}}(x, y, z)$ (resp. $\left.C_{\mathrm{F}}(x, y, z)\right)$ the colour values of the voxel located at the spatial coordinates $(x, y, z)$ for the initial (resp. final) configuration. After the conversion of raw images into binary images, the configurations are composed of voxels with two colour values only, namely, 0 (black) for voids and 255 (white) for glass beads. The colour value of the voxel located at the coordinates $(x, y, z), C_{\mathrm{R}}(x, y, z)$ of the compared configuration, is calculated from $C_{\mathrm{I}}$ and $C_{\mathrm{F}}$ from the following operation:

$$
C_{\mathrm{R}}(x, y, z)=\left|C_{\mathrm{I}}(x, y, z)-C_{\mathrm{F}}(x, y, z)\right|
$$

where | | represents the absolute value.

After this operation, the voxels affected by particle movement with colour changes from the initial configuration to the final configuration are coloured in white. However, the resulting black voxels can correspond to either the areas with movement or the areas without movement. A vertical cross section of the compared configuration corresponding to $\delta=0 \mathrm{~mm}\left(C_{\mathrm{I}}\right)$ and to $\delta=2$ $\mathrm{mm}\left(C_{\mathrm{F}}\right)$ is given in Fig. 9(a) as an example. In order to eliminate the small movements of the glass beads, due to the rotation and the translation of the sample during the scanning process, the compared configuration is eroded. In this process, each white area is reduced on its bound-

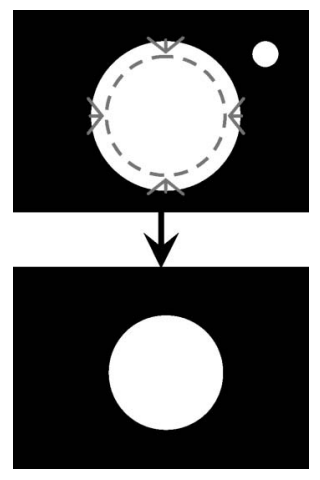

Fig. 10. Illustration of erosion process

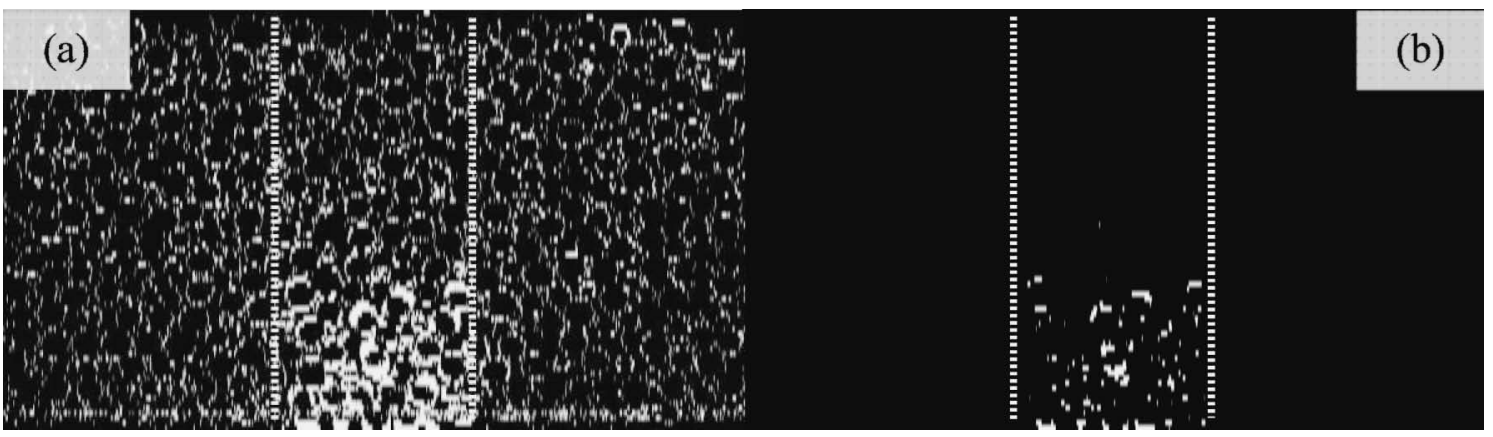

Fig. 9. Vertical cross section of compared configuration between $\delta=0 \mathrm{~mm}$ and $\delta=2 \mathrm{~mm}$ : a) raw compared configuration without erosion and b) compared configuration with erosion (vertical lines represent projection of trapdoor position) 
ary, as illustrated in Fig. 10. As a result, the smallest white areas that are not due to the trapdoor displacement are cancelled. The results of the erosion process are shown in Fig. 9(b).

In order to be able to compare the experimental results from the CT scanning to the numerical results from DEM, the same process of comparing configurations is used with the DEM results.

\section{Calculation of the Porosity}

In addition, the configuration corresponding to each displacement step, $\delta$, is analysed in terms of void ratio. Void ratios are calculated in restricted parts of the sample from the number of voxels and the threshold CT values, as explained in Eq. (9).

\section{COMPARISON OF X-RAY CT AND DEM RESULTS}

The arching phenomenon affects the redistribution of the forces occurring in the granular material. This effect is quantified by measuring the force applied on the trapdoor by the granular material during the tests. Arching also produces deformations in the granular layer, which are quantified by the evolution of the movements in the granular layer which are displacements and also by the loosening of the granular layer. These two aspects of the trapdoor mechanisms, namely, force and deformation in the granular layer, are presented in this section.

\section{Force Measurement}

The evolution of the force on the trapdoor as the trapdoor moved downward is given in Fig. 11 for the experimental and the numerical results. The vertical error bars represent the standard deviation obtained at the measurement point for each series of tests conducted under similar conditions.

The evolution of force on the trapdoor is very similar for the experimental tests and the numerical analysis. The arching effect develops as soon as the trapdoor is moved. The arching effect results in the report of a part of the

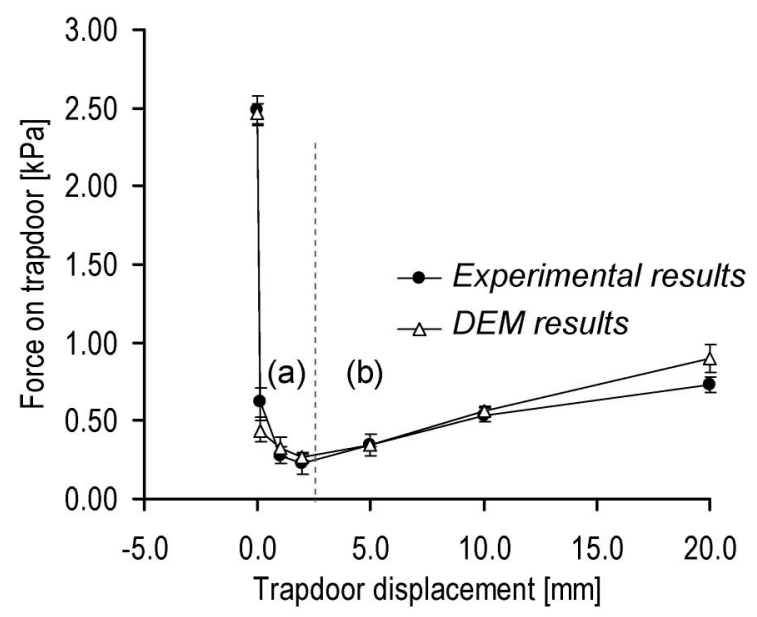

Fig. 11. Force on trapdoor versus trapdoor displacement $\delta$, for experimental and numerical results load initially applied on the trapdoor, to the granular mass located in the vicinity of the trapdoor. Consequently, as soon as the trapdoor is moved downward until $\delta=0.1 \mathrm{~mm}$, the force on the trapdoor decreases suddenly from its initial value due to the arching effect. A 75 $\%$ decrease in the experimental results and an $80 \%$ decrease in the numerical results are seen. The phase of force decrease continues until a trapdoor displacement of $\delta=2.0 \mathrm{~mm}$. The minimal force on the trapdoor is then very similar for both methods, namely, $0.22 \mathrm{~N}$ for the experimental tests and $0.26 \mathrm{~N}$ for the numerical tests. Then, as the trapdoor displacement increases $(\delta>2.0 \mathrm{~mm})$, the force on the trapdoor starts to increase until the end of the tests. For $\delta=5.0 \mathrm{~mm}$ and $\delta=10.0 \mathrm{~mm}$, the experimental and the numerical results are very similar. However, for $\delta=20.0 \mathrm{~mm}$, a difference can be noticed between the two results. The force on the trapdoor was smaller in the experimental tests than in the numerical analysis. This means that the load transfers due to the arching effect in the granular layer are stronger in the experimental tests for the condition of a relatively large displacement.

Despite a slight difference in behaviour between the experimental and the numerical conditions for the phase of increase in force, the reproduction of the experimental conditions with the numerical analysis is satisfying, especially considering the decrease in the phase of force. Until the trapdoor displacement reaches the value $\delta=10.0 \mathrm{~mm}$, the similarity in the results obtained with both methods is very good. The slight difference between the numerical and the experimental results for the biggest displacement steps may be due to the method used to move the trapdoor. In the experimental tests, the trapdoor was not really moved at once, because of having to move the screw. The real effect of moving the screw is that the trapdoor displacement can be compared to a succession of small movements. In the modelling, the trapdoor is really moved downward. This means that from one time step to the next (i.e., $5 \times 10^{-6}$ seconds later), the position of the trapdoor changed. This reason may explain why the difference occurred only at the end of the tests when the displacement increment is the biggest (an increment of 10 $\mathrm{mm}$ between $\delta=10$ and $\delta=20 \mathrm{~mm}$ ).

Some models were proposed to evaluate the part of the load applied on the trapdoor. Among these, Papamichos et al. (2001) suggested that minimal pressure on the trapdoor could be evaluated following a failure mechanism such as the one shown in Fig. 12(a) and extended to the three-dimensional square-shaped trapdoor shown in Fig. 12(b) and for which inclination $\beta$ is taken as being equal to $\beta=45^{\circ}-\varphi / 2$, with $\varphi$, the friction angle of the granular material. A simple comparison was made between the minimal pressure predicted by Papamichos et al. (2001) and the results obtained with the trapdoor tests performed in this study.

The balance of vertical forces in the failure zone implies that the whole weight of the failure zone is transmitted to the trapdoor, namely, load transfer $\mathrm{H}$ between the failure zone and the remaining granular material is only 


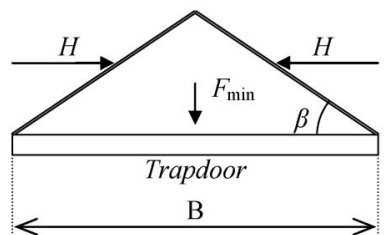

(a)

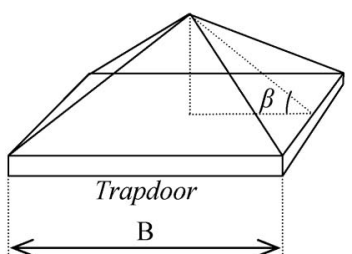

(b)
Fig. 12. Failure mechanism (a) proposed by Papamichos et al. (2001) for evaluation of minimal pressure on trapdoor in plane strain conditions and (b) extended to square-shaped trapdoor

horizontal. In the three-dimensional case, volume $\mathrm{V}$ of the failure zone can be calculated by the following equation:

$$
V=\frac{B^{3}}{2} \tan \left(45^{\circ}-\frac{\varphi}{2}\right),
$$

where $B$ is the trapdoor width and $\varphi$ is the friction angle of the granular material. Then, the minimal resulting force on the trapdoor, $F_{\min }$, is

$$
F_{\min }=\frac{\gamma B^{3}}{2} \tan \left(45^{\circ}-\frac{\varphi}{2}\right),
$$

where $\gamma=2.52 \mathrm{~g} / \mathrm{cm}^{3}$ is the density of the granular material.

Considering the peak friction angle of the granular material, Eq. (12) gives a value of minimal force $F_{\min }=$ $0.29 \mathrm{~N}$ with a volume $V_{\mathrm{f}}=18.5 \mathrm{~cm}^{3}$. The value of the predicted force is relatively close to the numerical and the experimental results. The real failure mechanism will be compared to the theoretical mechanism in the following section.

\section{Particle Displacements}

As the trapdoor is moved downward, the arching effect appears to redirect part of the weight of the granular material away from the trapdoor. A progressive release of vertical stress just above the trapdoor induces the rearrangement of the granular material. The local rearrangement of particles creates an expansion zone above which arching can develop. This expansion process starts in the vicinity of the trapdoor at the bottom of the granular layer and propagates towards the top surface of the granular layer, as the trapdoor displacement increases. From now on, the volume or the area of the granular material that expanded due to the trapdoor displacement is called the "expansion zone". The compared configurations (cf. Section "Binary Images") between two different displacement steps of the trapdoor are used to evaluate the propagation through the granular layer of the expansion zone due to the trapdoor displacement. Vertical cross sections of samples in the median and the diagonal axes of the trapdoor, shown in Fig. 13, are given for the different displacement steps of the trapdoor in Fig. 14 for the experimental results and Fig. 15 for the numerical results.

The cross sections showed progress in the expansion zone from the bottom of the layer towards the ground surface. The comparison of the expansion zones obtained
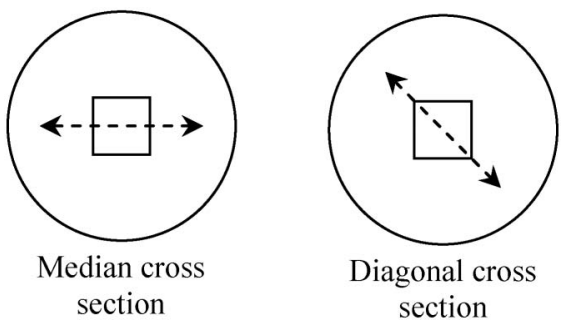

Fig. 13. Definition of cross sections

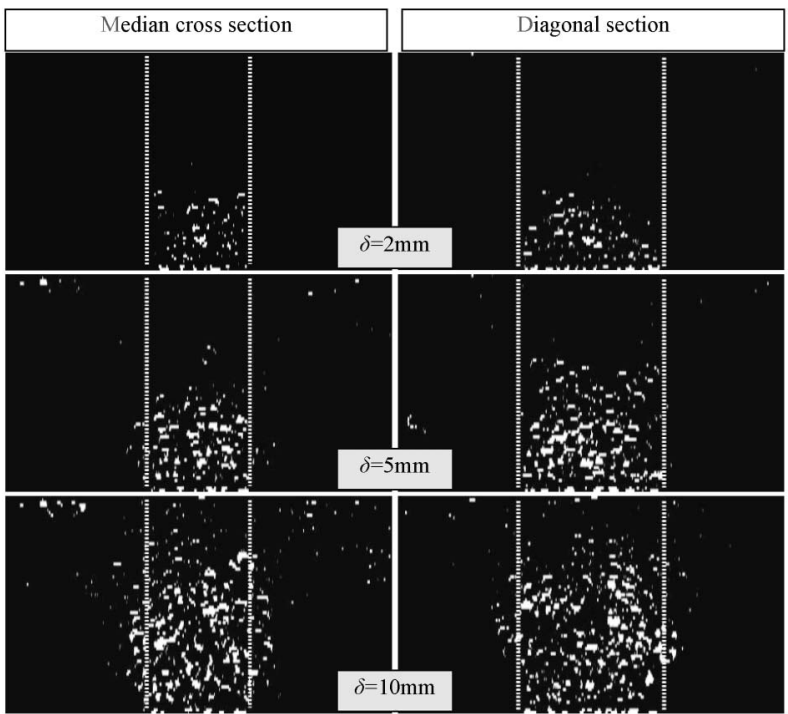

Fig. 14. Vertical cross sections of density change (from CT scanning) in median direction (left) and in diagonal direction (right) (vertical lines represent projection of trapdoor position)

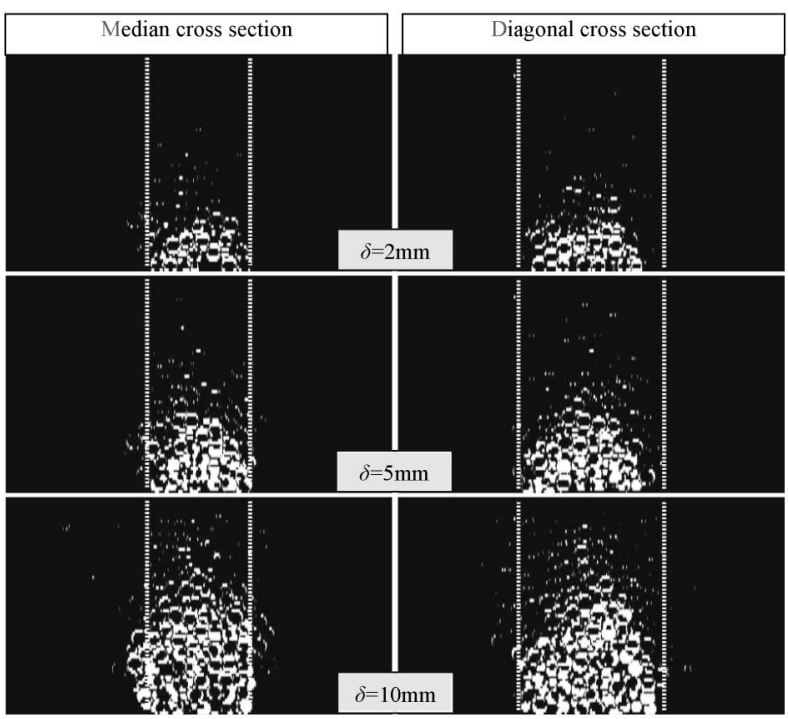

Fig. 15. Vertical cross sections of displacements (from DEM) in median direction (left) and in diagonal direction (right) (vertical lines represent projection of trapdoor position)

with the experimental and the numerical results was quantitatively fairly good. For a large trapdoor displacement $(\delta=10 \mathrm{~mm})$, it has to be noted that in the median 
axis cross sections, the expansion zone extended beyond the limit given by the trapdoor size, while it remained contained within the trapdoor size limit in the diagonal axis. As shown in Fig. 16, the horizontal cross sections of the expansion zone revealed a circular-shaped section despite the shape, even if the trapdoor was square.

In addition to the plane views, a three-dimensional reconstruction of the expansion zone was made using a series of horizontal cross sectional images. The threedimensional views are displayed in Fig. 17 and can calculate the volume of the granular material due to the arching affected by the trapdoor displacement. The expansion zones extracted from the experimental results and from the numerical results are found to be very similar not only
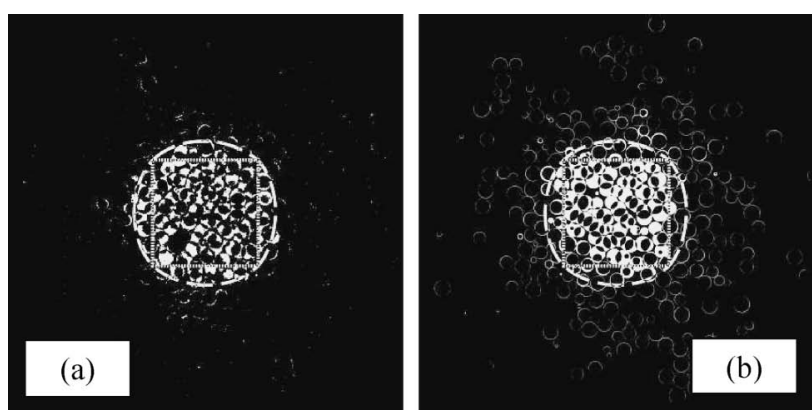

Fig. 16. Horizontal cross sections of compared configuration (between $0 \mathrm{~mm}$ and $10 \mathrm{~mm}$ ) for experimental sample (a) and numerical sample (b) (square line represents position of trapdoor) in shape, but also in volume (see Fig. 17). This good agreement can be obtained for all steps of the trapdoor displacement values, namely, $\delta=2,5$ and $10 \mathrm{~mm}$.

Considering the maximal load transfers in the granular layer obtained for $\delta=2 \mathrm{~mm}$, the minimal force on the trapdoor calculated with Eq. (12) was assumed to correspond to a volume of granular material, $V_{\mathrm{f}}=18.5 \mathrm{~cm}^{3}$. However, the volume of the expansion zone for $\delta=2 \mathrm{~mm}$ was equal to $V_{\mathrm{f}}=44.7 \mathrm{~cm}^{3}$ for the experimental tests. Despite the relatively good prediction given by Eq. (12), the weight of the expansion zone obtained in the numerical modelling was much larger than the force measured on the trapdoor. Therefore, the assumption of the horizontal direction of the load transfer between the expansion zone and the remaining granular material is not verified in the case presented here.

\section{Loosening Mechanism}

The void ratio of the sample was calculated for every horizontal cross section corresponding to a slice of the sample, $1 \mathrm{~mm}$ in thickness, from the white voxels corresponding to glass and the black voxels corresponding to air (see Fig. 8). The loosening of the granular layer above the trapdoor was evaluated from the distribution of void ratios for the sample in the height of the sample. In each horizontal cross section, the void ratio was calculated in an area corresponding to the circumcircle of the trapdoor represented in Fig. 16. Distributions of void ratio were calculated at each value of displacement for the trapdoor

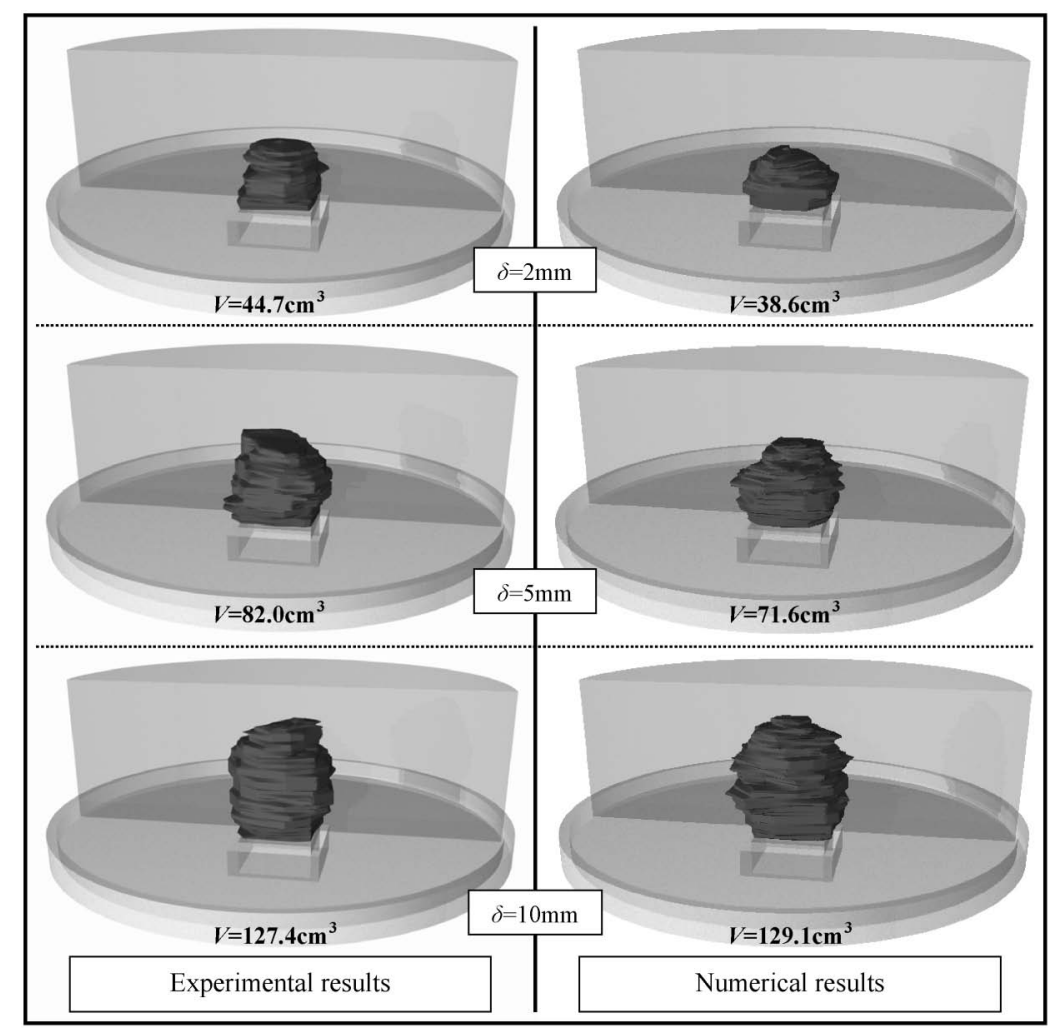

Fig. 17. Three-dimensional view of disturbed area for different displacement steps of trapdoor: experimental results (left) and numerical results (right) 

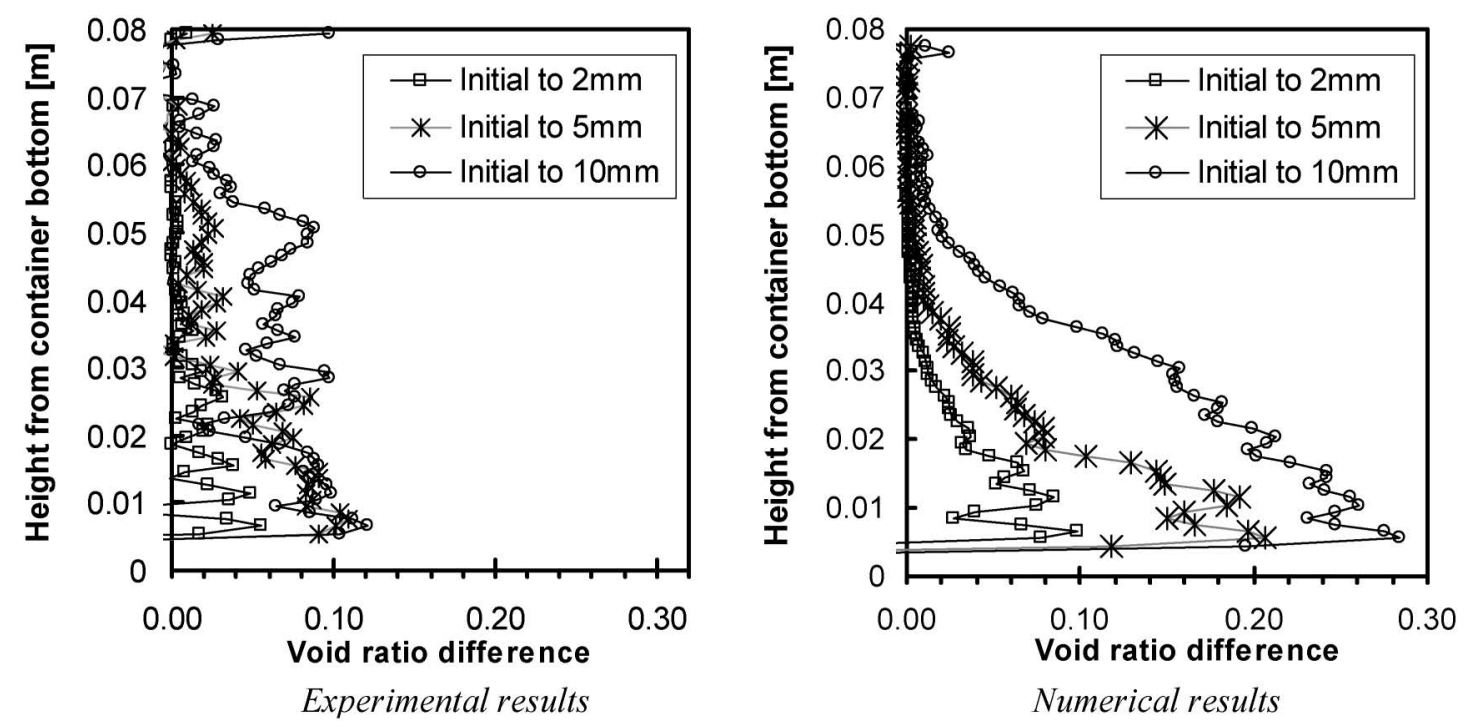

Fig. 18. Difference of void ratios in granular layer above trapdoor between initial state and different displacement steps of trapdoor: experimental results (left) and numerical modelling (right) (distribution corresponding to numerical results is average distribution of three different tests)

$(\delta=0,2,5$ and $10 \mathrm{~mm})$. Then, the initial state $(\delta=0 \mathrm{~mm})$ was subtracted from the following displacement step in order to obtain the loosening of the sample, called the "void ratio difference". The vertical distribution of the difference in void ratio in the height of the granular layer is given in Fig. 18.

The vertical distribution of the difference in void ratios confirms the progressive vertical expansion of the disturbed area as the displacement of the trapdoor increased. However, a difference in the loosening process can be noticed. In the experimental tests, the granular material progressively loosened until it reached the maximal void ratio of the glass beads, $e_{\max }=0.652$. The difference between maximal void ratio $e_{\max }$ and initial void ratio of the samples $e_{\min }$ is equal to 0.10 . Once the maximal void ratio was reached, the disturbed area then extended higher in the granular layer. For the numerical modelling, the granular material constantly loosened as the trapdoor displacement increased until a void ratio of $e=e_{\min }+$ 0.30 . Consequently, the loosening behaviour of the experimental material and the discrete elements might present some difference, due to the effect of dilatancy in the granular material.

\section{CONCLUSIONS}

A comparative study of the trapdoor problem under non-plane strain conditions, but in three dimensions, was conducted involving experimental tests and DEM modelling. From the experimental point of view, an X-ray CT was used in order to observe the kinematics. The use of $\mathrm{X}$-ray CT was very efficient for observing the local arching phenomena occurring in the layer of glass beads in three dimensions. A comparison of the different configurations of the sample allowed the monitoring of the effect disturbance created by the displacement of the trapdoor. The local loosening of the sample could also be calculat- ed. Besides these qualitative results, a force measurement apparatus was used to measure the intensity of the load transfers in the granular layer. In parallel, a numerical modelling of the experimental model test was developed using the Discrete Element Method. The numerical analysis reproduced exactly the tested material of the glass beads.

From a quantitative point of view, the intensities of the load transfers (force on the trapdoor) were very well reproduced by the numerical analysis. The first phase of force decrease was observed until a trapdoor displacement of $2 \mathrm{~mm}$, followed by a phase of force increase. The repeatability of the tests was also very good, since only a very small dispersion in the results was obtained. As far as the arching mechanisms were concerned, the disturbance due to trapdoor motion propagated in the granular layer, in the form of particle displacements, was precisely monitored in the experimental tests and very well reproduced by the DEM analysis. The volumes of the disturbed areas of the granular layer were very similar for each of the displacement steps studied. Considering the loosening of the disturbed areas, a slight difference in behaviour between the numerical and the experimental results was obtained for larger displacement levels. In the numerical results, the granular material seemed to be able to loosen beyond the experimental maximal void ratio.

Now that the testing procedure for the non-plane strain conditions has been validated based on the use of an Xray $\mathrm{CT}$, attention will be focused on the effects of the size of the particles and the shape of the trapdoor on the arching mechanisms. Furthermore, in order to extend the results of this study to the field of geomechanics, and thus, to real soils, other particle size distributions will be considered by mixing several particle sizes.

\section{ACKNOWLEDGMENTS}


This research was conducted with a Grant-in-Aid from the Japanese Society for the Promotion of Science (JSPS 20.08818).

\section{LIST OF NOTATIONS}

\section{$C T_{\mathrm{i}} \quad$ CT value of class $\mathrm{i}$}

$C_{\mathrm{F}}(x, y, z)$ colour value of voxel located at coordinates $(x, y, z)$ in final configuration

$C_{\mathrm{I}}(x, y, z) \quad$ colour value of voxel located at coordinates $(x, y, z)$ in initial configuration

$C_{\mathrm{R}}(x, y, z)$ colour value of voxel located at coordinates $(x, y, z)$ in compared configuration

$d \quad$ mean diameter of particles $(\mathrm{m})$

e void ratio

$e_{\min } \quad$ minimal void ratio

$e_{\max } \quad$ maximal void ratio

$F \quad$ force on trapdoor $(\mathrm{N})$

$F_{\min } \quad$ minimal force on trapdoor $(\mathrm{N})$

$f_{\mathrm{n}} \quad$ normal component of contact force between particles (N)

$f_{\mathrm{t}} \quad$ tangential component of contact force between particles (N)

$H \quad$ load transfer force between failure zone and remaining part of layer $(\mathrm{N})$

$k_{\mathrm{n}} \quad$ normal stiffness of contact between particles $(\mathrm{N} / \mathrm{m})$

$k_{\mathrm{t}} \quad$ tangential stiffness of contact between particles $(\mathrm{N} / \mathrm{m})$

$n_{\mathrm{i}} \quad$ number of voxels in class i of CT value

$P \quad$ mean isotropic pressure in particle sample $(\mathrm{Pa})$

$u_{\mathrm{t}} \quad$ relative tangential displacement between two particles in contact $(\mathrm{m})$

$v \quad$ volume of single voxel $\left(\mathrm{m}^{3}\right)$

$V_{\mathrm{a}} \quad$ volume of air $\left(\mathrm{m}^{3}\right)$

$V_{\mathrm{g}} \quad$ volume of glass beads $\left(\mathrm{m}^{3}\right)$

$V_{\mathrm{t}} \quad$ total volume (glass beads and air) $\left(\mathrm{m}^{3}\right)$

$\beta \quad$ inclination with horizontal of failure zone for maximal load transfer phase (degree)

density of glass beads $\left(\mathrm{g} / \mathrm{cm}^{3}\right)$

trapdoor vertical displacement $(\mathrm{mm})$

overlap distance between two particles in contact (m)

non-dimensional stiffness level

friction coefficient of contact between particles

absorption coefficient of $\mathrm{x}$-ray beam

absorption coefficient of $\mathrm{x}$-ray beam for water

friction angle (degree)

peak friction angle (degree)

critical friction angle (degree)

\section{REFERENCES}

1) Chareyre, B. and Villard, P. (2005): Dynamic spar elements and DEM in two dimensions for the modelling of soil-inclusion prob- lems, Journal of Engineering Mechanics, ASCE, 131(7), 689-698.

2) Chevalier, B., Combe, G. and Villard, P. (2009): Experimental and numerical study of the response of granular layer in the trap-door problem, Powders and Grains 2009: Proc. of the 6th Int. Conf. on Micromechanics of Granular Media, Springer, 649-652.

3) Combe, G. (2002): Mécanique des matériaux granulaires et origines microscopiques de la déformation, Etudes et Recherches du Laboratoire Central des Ponts et Chaussées.

4) Cundall, P. A. and Strack, O. D. L. (1979): A discrete numerical model for granular assemblies, Géotechnique, 29, 47-65.

5) Dewoolkar, M. M., Santichaianant, S. and Ko, H-Y. (2007): Centrifuge modelling of granular soil response over active circular trapdoors, Soils and Foundations, 47(5), 931-945.

6) Handy, R. L. (1985): The arch in soil arching, Journal of Geotechnical Engineering, 111(3), 302-318.

7) Koutsabeloulis, N. C. and Griffiths, D. V. (1989): Numerical modeling of the trap-door problem, Géotechnique, 39(1), 77-89.

8) Magnier, S. A., and Donzé, F. V. (1997): Discrete element project, Technical report, Université du Québec-Montréal.

9) Otani, J., Mukunoki, T. and Obara, Y. (2000): Application of Xray $\mathrm{CT}$ method for characterization of failure in soils, Soils and Foundations, 40(2), 111-118.

10) Otani, J., Mukunoki, T. and Obara, Y. (2002): Characterization of failure in sand under triaxial compression using an industrial X-ray CT scanner, Journal of Physical Modelling in Geotechnics, 1, $15-22$.

11) Papamichos, E., Vardoulakis, I. and Heil, L. K. (2001): Overburden modelling over a compacting reservoir using a trap-door apparatus, Physic and Chemistry of Earth (A), 26(1-2), 69-74.

12) Schäfer, S., Dippel, S. and Wolf, D. (1996): Forces schemes in simulations of granular materials, Journal de physique I, 6(1), $5-20$.

13) Tanaka, T. and Sakai, T. (1993): Progressive failure and scale effect of trap-door problems with granular materials, Soils and Foundations, 33(1), 11-22.

14) Terzaghi, K. (1936): Stress distribution in dry and saturated sand above a yielding trapdoor, Proceedings of International Conference of Soil Mechanics, Cambridge University, 1, 307-311.

15) Terzaghi, K. (1943): Theoretical Soil Mechanics, New-York, John Wiley and Sons.

16) Thornton, C. and Sun, G. (1993): Axisymetric compression of 3D polydisperse systems of spheres, Powders and Grains 93: Proc. of the 2nd Int. Conf. on Micromechanics of Granular Media, Balkema, 129-134.

17) Vardoulakis, I., Graf, B. and Gudehus, G. (1981): Trap-door problem with dry sand: a statical approach based upon model test kinematics, International Journal for Numerical and Analytical Methods in Geomechanics, 5, 57-78.

18) Vardoulakis, I., Vairaktaris, E. and Papamichos, E. (2004): Subsidence diffusion-convection: I. The direct problem, Computational Methods in Applied Mechanics and Engineering, 193, 2745-2760. 